\begin{tabular}{|c|c|}
\hline Title & Quantitative analysis of helium by post-ionization method using femtosecond laser technique \\
\hline Author(s) & $\begin{array}{l}\text { Yurimoto, Hisayoshi; Bajo, Ken-ichi; Sakaguchi, Isao; Suzuki, T aku T.; Jurewicz, A my J. G.; Itose, Satoru; Uchino, } \\
\text { Kiichiro; Ishihara, Morio }\end{array}$ \\
\hline Citation & $\begin{array}{l}\text { Surface and interface analysis, 48(11), 1181-1184 } \\
\text { https://doi.org/10.1002/sia.6119 }\end{array}$ \\
\hline Issue Date & $2016-11$ \\
\hline Doc URL & http:/hdl.handle.net/2115/67013 \\
\hline Rights & $\begin{array}{l}\text { This is the peer reviewed version of the following article: Y urimoto, H., Bajo, K., Sakaguchi, I., Suzuki, T. T., } \\
\text { Jurewicz, A. J. G., Itose, S., U Chino, K., and Ishihara, M. (2016) Quantitative analysis of helium by post-ionization } \\
\text { method using femtosecond laser technique. Surf. Interface A nal., 48: 1181-1184, which has been published in final } \\
\text { form at doi:10.1002/sia.6119. This article may be used for non-commercial purposes in accordance with Wiley Terms } \\
\text { and Conditions for Self-A rchiving. }\end{array}$ \\
\hline Type & article (author version) \\
\hline File Information & Quantitative analysis.pdf \\
\hline
\end{tabular}

Instructions for use 


\section{Quantitative analysis of helium by post-ionization method using femto-second laser technique}

Hisayoshi Yurimoto ${ }^{1,2 *}$, Ken-ichi Bajo ${ }^{1}$, Isao Sakaguchi ${ }^{3}$, Taku T. Suzuki ${ }^{3}$, Amy J. G. Jurewicz ${ }^{4}$, Satoru Itose ${ }^{5}$, Kiichiro Uchino $^{6}$ and Morio Ishihara ${ }^{7}$

${ }^{1}$ Department of Natural History Sciences, Hokkaido University, IIL, Sapporo 001-0021, Japan

${ }^{2}$ Institute of Space and Astronautical Science, Japan Aerospace Exploration Agency, Sagamihara, Kanagawa 252-5210, Japan

${ }^{3}$ National Institute for Materials Science, Tsukuba, Ibaraki 305-0044, Japan

${ }^{4}$ CMS/SESE, Arizona State University, Tempe, AZ 85287-1404, U.S.A.

5 JEOL Ltd., Musashino, Akishima, Tokyo 196-8558, Japan

${ }^{6}$ Graduate School of Engineering Sciences, Kyushu University, Kasuga, Fukuoka 816-8580, Japan

${ }^{7}$ Department of Physics, Osaka University, Toyonaka, Osaka 560-0043, Japan

*Corresponding author: yuri@ep.sci.hokudai.ac.jp

Abstract: Helium has the largest ionization potential of all elements; thus, it is difficult to ionization for measurement by mass spectrometry. In order to analyze He, a tunnel-ionization time-of-flight sputtered neutral mass spectrometry system (called LIMAS) has recently been developed. LIMAS uses a femto-second laser technique, and can ionize He to achieve 10\% ionization yields [1]. We quantified the effectiveness of this method for He analysis from a $2.5 \times 4$ $\mu^{2}$ area of He-implanted silicon. The amount of He in a implant was quantified by measuring the ion-current, giving a nominal implant fluence per unit area. Thus, the fraction of total He measured by LIMAS during depth profiling could be quantified by comparison with the He-concentration of the reference implant. The $\mathrm{He}^{+}$intensities normalized by host ions of Si linearly correlated with the known He concentrations with a reproducibility of $10 \%$ at concentrations less than $10^{21} \mathrm{~cm}^{-3}$. The detection limit was down to $10^{18} \mathrm{He}^{-3}$ (20 ppm). For concentrations exceeding $10^{21} \mathrm{~cm}^{-3}$, the He intensities are smaller than those expected from the lower concentration range. This non-linearity may reflect the limit of retention of He in the Si lattice, since He is chemically inert. 


\section{Introduction}

Reliable, quantitative depth profiles for He are highly desirable for materials used in nuclear reactions and fusion physics [2] as well as for extraterrestrial materials irradiated by solar wind and cosmic rays [3]. Secondary ion mass spectrometry (SIMS) is considered to be an extremely sensitive micro-area analytical technique, which can provide elemental depth-profiles of solid surfaces [4]. Quantification of $\mathrm{He}$ is often difficult, however, because of the extremely low secondary-ionization yields resulting from its high ionization energy [2, 5, 6]. Recently, sputtered neutral mass spectrometry (SNMS) was successfully used to measure the depth-profile distribution of solar wind helium from a NASA Genesis target [3]. They used strong-field tunnel ionization to detect He neutrals sputtered from a $\sim 1 \mathrm{~m}^{2}$ area of the surface.

In this study, we studied the possibility of He quantification in the laboratory using SNMS with tunneling ionization for analyzing He ion-implanted silicon substrates.

\section{Experimental}

This study used silicon wafer substrates containing ${ }^{4} \mathrm{He}^{+}$implanted at energies of: (i) $15 \mathrm{keV}$ with a nominal fluence of $1 \times 10^{16} \mathrm{~cm}^{-2}$ (Leonard Kroko, Inc.); (ii) $27 \mathrm{keV}$ with nominal fluences of $0.5,0.8,2$, and $8 \times 10^{16} \mathrm{~cm}^{-2}$; and (iii) $4 \mathrm{keV}$ with a nominal fluence of $1 \times 10^{15} \mathrm{~cm}^{-2}$. The ion currents are $50 \mathrm{nA}$.

An SNMS instrument called LIMAS [1, 7] was used for our measurements. Operation timings of the measurement sequence for LIMAS were described elsewhere [8]. A pulsed primary beam of ${ }^{69} \mathrm{Ga}$ (30 keV and $38 \mathrm{nA}$ ) was focused to a spot $800 \mathrm{~nm}$ in diameter on the surface of the Si wafer. The pulse period was set to 500 ns. Sputtered neutrals were ionized by a focused ( $50 \mu \mathrm{m}$ diameter at $100 \mu \mathrm{m}$ above the sputtered points) femtosecond laser beam (wavelength: $800 \pm 60 \mathrm{~nm}$ ) under strong-field ionization. The laser pulse was $40 \mathrm{fs}$ with an energy of $3 \mathrm{~mJ}$ and a $1 \mathrm{kHz}$ repetition. 
Since the laser power density of the post-ionization exceeded $10^{-19} \mathrm{~W} \mathrm{~m}^{-2}$, the ionization corresponds to tunneling mechanisms [1]. We accumulated post-ionized ions for 200 primary beam pulses at each spot to collect data as a function of depth, allowing us to discriminate He depth distributions. The primary beam was rastered to form a crater in a sampling pattern of $15 \times 15$ spots with a step interval of $500 \mathrm{~nm}$. Each crater had an area of $8 \times 13 \mu \mathrm{m}^{2}$ on the surface because the incident angle of the primary beam (formed by the optical axis of the primary beam on the surface and the normal to the surface at the point of incidence) was set to $55^{\circ}$. At this angle, the sample surface became perpendicular to the optical axis of the ion extraction optics into the mass spectrometer.

Positive ions were introduced into a multi-turn time-of-flight mass spectrometer [9] by an acceleration voltage of $-4 \mathrm{kV}$ with $1 \mathrm{kHz}$ repetition (synchronized with the primary ion pulses). Measurements were conducted for all the rastered primary beam spots, and only ions generated from the center $\left(2.5 \times 4 \mu^{2}, 5 \times 5\right.$ spots $)$ of the sputtered craters were used to determine the depth profiles in order to avoid crater edge effects. The flight path length of the mass spectrometer was set to $68 \mathrm{~m}$ for ${ }^{4} \mathrm{He}^{+}$. We measured ${ }^{4} \mathrm{He}^{+},{ }^{28} \mathrm{Si}^{4+},{ }^{28} \mathrm{Si}^{3+},{ }^{28} \mathrm{Si}^{2+}$, and ${ }^{28} \mathrm{Si}^{+}$. The intensities of each mass were integrated with respect to its time-of-flight mass spectrum to calculate the respective ion intensities. The ${ }^{4} \mathrm{He}^{+}$intensities were normalized to individual or the total intensities of ${ }^{28} \mathrm{Si}^{4+},{ }^{28} \mathrm{Si}^{3+},{ }^{28} \mathrm{Si}^{2+}$, and ${ }^{28} \mathrm{Si}^{+}$and translated to concentrations using their fluences. The vacuum of the sample chamber was maintained at $3 \times 10^{-8} \mathrm{~Pa}$ during measurements. The depths of sputtered craters were measured after depth profiling analyses by an atomic force microscope (Asylum Technology, MFP-3D-BIO-J). 


\section{Results and Discussion}

A typical depth profile of ${ }^{4} \mathrm{He}^{+}$implanted in the Si wafer is shown in Fig. 1. The intensities of Si ions appear constant during analysis, indicating a stable sputtering rate and stable post-ionization. Therefore, we assume a constant sputter rate when converting time to depth for each measurement and adopt an average sputtering rate determined by dividing the total crater depth by the total sputter time. The ${ }^{4}$ He profiles strongly suggest an ion implant, but a certain background always remains (i.e., the signal never drops to zero). This ${ }^{4} \mathrm{He}$ background can also be observed for samples without ion implants under the same measurement conditions. The similar intensities seen for all samples indicate that this blank is attributable to the by ${ }^{4} \mathrm{He}$ gas in the photoionization volume above the sample surface. The ions comprising the blank come from the residual He in the vacuum and from adsorbed He on the surface sputtered by the primary beam. Note that the sample chamber was maintained at an ultrahigh vacuum of $3 \times 10^{-8} \mathrm{~Pa}$.

The concentration profiles for He implanted into silicon wafers were calculated by the TRIM code [10] for each implant energy, then the maximum concentrations at the implant peak were calculated using the nominal implant fluence. The maximum concentrations of He increase with implantation fluences of He for the experiments in this study (Fig. 2). On the other hand, the maximum values of the ${ }^{4} \mathrm{He}^{+} /{ }^{28} \mathrm{Si}^{4+}$ ratio observed in the depth profile by LIMAS do not monotonically increase with He fluences. The ratios linearly increase with fluence up to $15 \times 10^{15}$ He $\mathrm{cm}^{-2}$, and abruptly decrease at around $20 \times 10^{15} \mathrm{He} \mathrm{cm}^{-2}$. The ratios increase again with the fluences beyond $20 \times 10^{15} \mathrm{He}^{-2}$, but the increasing rate becomes small. The abrupt decrease suggests that the peak concentration of He implanted at that $\mathrm{keV}$ and fluence exceeded a margin for the He to hold in silicon crystal.

For depth profiles at fluences less than $15 \times 10^{15} \mathrm{He} \mathrm{cm}^{-2}$, the ion intensity ratios of ${ }^{4} \mathrm{He}^{+} /{ }^{28} \mathrm{Si}^{4+}$ from the ion-implanted Si sample were converted to concentrations (atoms $\mathrm{cm}^{-3}$ ) by 
normalizing the model calculated by the TRIM code to the nominal ion fluence. That is, the integral of the measured depth distribution was equated to the fluence of the implant. The general shapes of the He implants measured experimentally are in excellent accordance with the TRIM models for He concentrations above the blank level (Fig. 3). This shows that the ${ }^{4} \mathrm{He}^{+} /{ }^{28} \mathrm{Si}^{4+}$ ratios are proportional to the respective concentrations and can be used for He quantification. The detection limit of He (defined by the background level) was down to $10^{18}$ atom $\mathrm{cm}^{-3}$. The detection limit in this study was similar to that previously reported [11], but the spatial resolution was much improved $(2.5 \times 4$ $\left.\mu \mathrm{m}^{2}\right)$.

Relative sensitivity factors (RSFs) determined for the implants having fluences less than $15 \times$ $10^{15} \mathrm{He} \mathrm{cm}^{-2}$ are given in Table1. Included in the Table 1 are the RSFs for ${ }^{4} \mathrm{He}$ referenced ${ }^{28} \mathrm{Si}^{4+},{ }^{28} \mathrm{Si}^{3+},{ }^{28} \mathrm{Si}^{2+},{ }^{28} \mathrm{Si}^{+}$, and $\Sigma^{28} \mathrm{Si}\left(={ }^{28} \mathrm{Si}^{4+}+{ }^{28} \mathrm{Si}^{3+}+{ }^{28} \mathrm{Si}^{2+}+{ }^{28} \mathrm{Si}^{+}\right)$, respectively. Reproducibility (mean, standard deviation) is given for results from both (1) implants having different fluences and implantation energies measured sequentially in a single session, and (2) the same sample measured on different dates. The RSFs are variable, ranging from $8 \%$ to $40 \%$ depending on the reference elements. In the both cases, however, the reproducibility of the RSF is better when ${ }^{28} \mathrm{Si}^{4+}$ or ${ }^{28} \mathrm{Si}^{3+}$ is used for the normalization than when ${ }^{28} \mathrm{Si}^{2+}$ or ${ }^{28} \mathrm{Si}^{+}$is used. The efficiencies of post-ionization of LIMAS strongly depend on both laser power density and ionization energy [1, 12]. Therefore, the differences in reproducibility may be associated with ionization volumes in the neutral plume, which are strongly depend on their ionization energy. The first ionization energy of He is $24.59 \mathrm{eV}$ which is higher than ionization energies of $\mathrm{Si}^{+}{\text {and } \mathrm{Si}^{2+}}^{2}$ and lower than those of $\mathrm{Si}^{3+}$ and $\mathrm{Si}^{4+}$. The results suggest that if the reference ion selected for normalizing the He has an ionization energy higher than $24.59 \mathrm{eV}$, then the quantitative analysis becomes more reproducible.

We may infer He ionization yield from the RSF referenced $\Sigma^{28} \mathrm{Si}$ for LIMAS. The average RSF of $7 \times 10^{22}$ atom $\mathrm{cm}^{-3}$ suggests that the He ionization yield achieve $70 \pm 15 \%$ under the 
condition in this study if ionization yield of Si is $100 \%$ [1] and useful yields of He and Si are the same.

Under experimental conditions with He fluence larger than $15 \times 10^{15} \mathrm{He} \mathrm{cm}^{-2}$, it appears that the implanted He escapes from the silicon substrate before the measurement (Fig. 2). Surprisingly, depth distribution after the partial He escape is similar to the as-implanted profile. The distributions of ${ }^{4} \mathrm{He}$ are apparently simulated by the TRIM code assuming applicable fluence to fit the profile (Fig. 4). The TRIM profiles resemble the observed depth profiles, but the simulated fluence is more than one order of magnitude smaller than the experimental one. Furthermore, the degree of fitness between measured and simulated profiles is less adapted compared with the relations shown in Fig. 3, especially for profiles at the surface side. These results show that we must carefully select standards of appropriate ion implantation conditions for the quantification of He concentrations.

The depth distribution shown in Fig. 4 suggests that the escape of He from the Si substrate cannot explained by a simple diffusion mechanism in uniform matter. This phenomenon rather resembles a sudden escape of He from the Si lattice due to exceeding the retention limit and effusion through the defect network generated by He ion implantation. Formation of nanometric bubbles may be related to this phenomenon. The minimal fluence required to observe helium bubbles in the as-implanted silicon is $5-10 \times 10^{15} \mathrm{He} \mathrm{cm}^{-2}$, and the minimal He peak concentration is $3.5 \times 10^{20} \mathrm{~cm}^{-3}[13]$. These values are slightly smaller than the value for He escape in this study, suggesting that connection of defects to form a network may effectively facilitate the He escape. For the He ion implantation experiments, connection of defects into a network may significantly develop above a fluence of $10 \times 10^{15} \mathrm{He} \mathrm{cm}^{-2}$ and facilitate the effusion of He from the Si wafer. The discordance of the surface side depth profiles in Fig. 4 suggests that the effusion of He occurs easier in the region beneath the surface. Effective He effusion is expected to occur abruptly when the network of defects connects to the surface of the Si wafer and is expected to periodically or gradually continue after the connection is established. Similar escape features for the change of He 
depth distribution from the as-implanted $\mathrm{He}$ profile in the $\mathrm{Si}$ wafer have been observed in isothermal He desorption experiments [14].

\section{Conclusions}

We have developed a method for quantitative analysis of He in a silicon substrate. A SNMS technique with strong-field post-ionization was applied to the depth profile analysis of a $2.5 \times 4$ $\mu \mathrm{m}^{2}$ area. Ion implantation into $\mathrm{Si}$ substrates was used to prepare standards with known concentration. A 10\% precision of He quantification reproducibility was achieved, and the detection

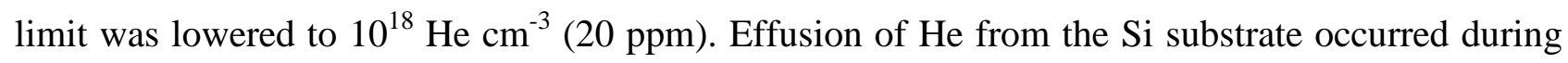
implantation experiments at fluences above $15 \times 10^{15} \mathrm{He}^{-2}$. The He effusion reduced the He concentration in the substrates, but the relative distribution remained largely original.

\section{Acknowledgements}

We thank Yoshikazu Fujii and two anonymous reviewers for their constructive comments. This work is partly supported by Monka-sho grants.

\section{References}

[1] S. Ebata, M. Ishihara, K. Uchino, S. Itose, M. Matsuya, M. Kudo, K.-i. Bajo, H. Yurimoto, Development of laser ionization mass nanoscope (LIMAS), Surf. Interface Anal., 44 (2012) 635-640. [2] H. Lefaix-Jeuland, S. Moll, F. Legendre, F. Jomard, SIMS depth profiling of implanted helium in pure iron using $\mathrm{CsHe}^{+}$detection mode, Nuclear Instruments and Methods in Physics Research Section B: Beam Interactions with Materials and Atoms, 295 (2013) 69-71.

[3] K.-i. Bajo, C.T. Olinger, A.J.G. Jurewicz, D.S. Burnett, I. Sakaguchi, T. Suzuki, S. Itose, M. Ishihara, K. Uchino, R. Wieler, H. Yurimoto, Depth profiling analysis of solar wind helium collected in diamond-like carbon film from Genesis, Geochem. J., 49 (2015) 559-566.

[4] A. Benninghoven, F.G. Rudenauer, H.W. Werner, Secondary Ion Mass Spectrometry: Basic Concepts, Instrumental Aspects, Applications and Trends (Chemical Analysis: A Series of Monographs on Analytical Chemistry and Its Applications), Wiley-Interscience, 1987. 
[5] H. Gnaser, H. Oechsner, Noval detection scheme for the analysis of hydrogen and helium by secondary ion mass spectrometry, Surf. Interface Anal., 17 (1991) 646-649.

[6] A.K. Tyagi, K.G.M. Nair, K. Krishan, SIMS depth profiling of implanted helium in Al-Mn alloy using CsHe+ molecular ion detection, Bulletin of Materials Science, 16 (1993) 45-49.

[7] A. Tonotani, K.-i. Bajo, S. Itose, M. Ishihara, K. Uchino, H. Yurimoto, Evaluation of multi-turn time-of-flight mass spectrum of laser ionization mass nanoscope, Surf. Interface Anal., (2015) This isuue.

[8] K.-i. Bajo, H. Yurimoto, Surf. Interface Anal., (2015) this issue.

[9] D. Okumura, M. Toyoda, M. Ishihara, I. Katakuse, A compact sector-type multi-turn time-of-flight mass spectrometer 'MULTUM II', Nuclear Instruments and Methods in Physics Research A, 519 (2004) 331-337.

[10] J.F. Ziegler, J.P. Biersack, M.D. Ziegler, SRIM, the stopping and range of ions in matter, Lulu Press Co., http://www.srim.org, 2012.

[11] R.G. Wilson, V.R. Deline, C.G. Hopkins, Depth distributions and range parameters for He implanted in Si and GaAs, Appl. Phys. Lett., 41 (1982) 929-931.

[12] R. Mibuka, S. Hassaballa, K. Uchino, H. Yurimoto, R. Todokoro, K. Kumondai, M. Ishihara, Characteristics of post-ionization using a femto-second laser, Appl. Surf. Sci., 255 (2008) 1595-1598. [13] V. Raineri, P.G. Fallica, G. Percolla, A. Battaglia, M. Barbagallo, S.U. Campisano, Gettering of metals by voids in silicon, Journal of Applied Physics, 78 (1995) 3727-3735.

[14] F. Corni, G. Calzolari, S. Frabboni, C. Nobili, G. Ottaviani, R. Tonini, G.F. Cerofolini, D. Leone, M. Servidori, R.S. Brusa, G.P. Karwasz, N. Tiengo, A. Zecca, Helium-implanted silicon: A study of bubble precursors, Journal of Applied Physics, 85 (1999) 1401-1408. 
Table 1. Relative sensitivity factor of ${ }^{4} \mathrm{He}$ implanted in silicon substrate.

\begin{tabular}{|c|c|c|c|c|c|}
\hline Reference mass & ${ }^{28} \mathrm{Si}^{i+}$ & ${ }^{28} \mathrm{Si}^{2+}$ & ${ }^{8} \mathrm{Si}^{3+}$ & $\mathrm{Si}^{4+}$ & $\Sigma^{28} \mathrm{~S}$ \\
\hline Ionization energy /eV & 8.15 & 16.35 & 33.49 & 45.14 & \\
\hline \multicolumn{6}{|c|}{ Ion implantation (on $2014 / 3 / 25$ ) } \\
\hline Energy Fluence & \multicolumn{5}{|c|}{ Relative sensitivity factor $/ 10^{22}$ atom $\mathrm{cm}^{-3}$} \\
\hline $15 \mathrm{keV} 1 \times 10^{16} \mathrm{~cm}^{-2}$ & 1.98 & 2.51 & 2.55 & 1.58 & 8.63 \\
\hline $27 \mathrm{keV} \quad 5 \times 10^{15} \mathrm{~cm}^{-2}$ & 1.15 & 1.33 & 1.94 & 1.44 & 5.85 \\
\hline $27 \mathrm{keV} \quad 8 \times 10^{15} \mathrm{~cm}^{-2}$ & 1.53 & 2.13 & 2.42 & 1.35 & 7.44 \\
\hline average & 1.55 & 1.99 & 2.30 & 1.46 & 7.31 \\
\hline standard deviation & $27 \%$ & $30 \%$ & $14 \%$ & $8 \%$ & $19 \%$ \\
\hline \multicolumn{6}{|c|}{ Ion implantation (at $27 \mathrm{keV}, 5 \times 10^{15} \mathrm{~cm}^{-2}$ ) } \\
\hline Date & \multicolumn{5}{|c|}{ Relative sensitivity factor $/ 10^{22}$ atom $\mathrm{cm}^{-3}$} \\
\hline $2014 / 3 / 12$ & 2.35 & 2.84 & 2.66 & 1.57 & 9.44 \\
\hline $2014 / 3 / 18$ & 2.52 & 3.80 & 2.45 & 1.82 & 10.6 \\
\hline $2014 / 3 / 19$ & 2.95 & 3.78 & 2.11 & 1.56 & 10.4 \\
\hline $2014 / 3 / 25$ & 1.15 & 1.33 & 1.94 & 1.44 & 5.85 \\
\hline $2014 / 3 / 26$ & 1.23 & 2.23 & 2.47 & 1.62 & 7.56 \\
\hline average & 2.04 & 2.80 & 2.33 & 1.60 & 8.77 \\
\hline dard deviation & $40 \%$ & $38 \%$ & - & - & $23 \%$ \\
\hline
\end{tabular}




\section{Figure Captions}

Figure 1. Ion intensity distribution measured as a function of depth (nm) from surface of a Si wafer implanted with $27 \mathrm{keV}^{4} \mathrm{He}^{+}$at a fluence of $5 \times 10^{15}$ ions $\mathrm{cm}^{-2}$. The intensities of various Si reference ions are nearly constant with depth, demonstrating stability.

Figure 2. Correlations between $\mathrm{He}^{+} / \mathrm{Si}^{4+}$ depth profile maximum values, maximum concentrations of implanted He ions simulated by TRIM, and their He fluences. Red symbols: $\mathrm{He}^{+} / \mathrm{Si}^{4+}$, blue symbols: TRIM.

Figure 3. Depth distributions of He implanted into Si wafers at 15 and $27 \mathrm{keV}$ with respective fluences shown in the figure. Solid curves were obtained using the TRIM code for each experimental condition.

Figure 4. Depth distributions of He implanted into Si wafers at $27 \mathrm{keV}^{4} \mathrm{He}^{+}$with experimental fluences of $2 \times 10^{16}$ (red) and $8 \times 10^{16}$ (blue) ions $\mathrm{cm}^{-2}$. Solid curves were obtained using the TRIM code for $27 \mathrm{keV}^{4} \mathrm{He}^{+}$with $2.5 \times 10^{15}$ (red) and $5 \times 10^{15}$ (blue) ions $\mathrm{cm}^{-2}$. Note deviation of the measured concentration from the expected concentration close to the radiation-damaged surface. 


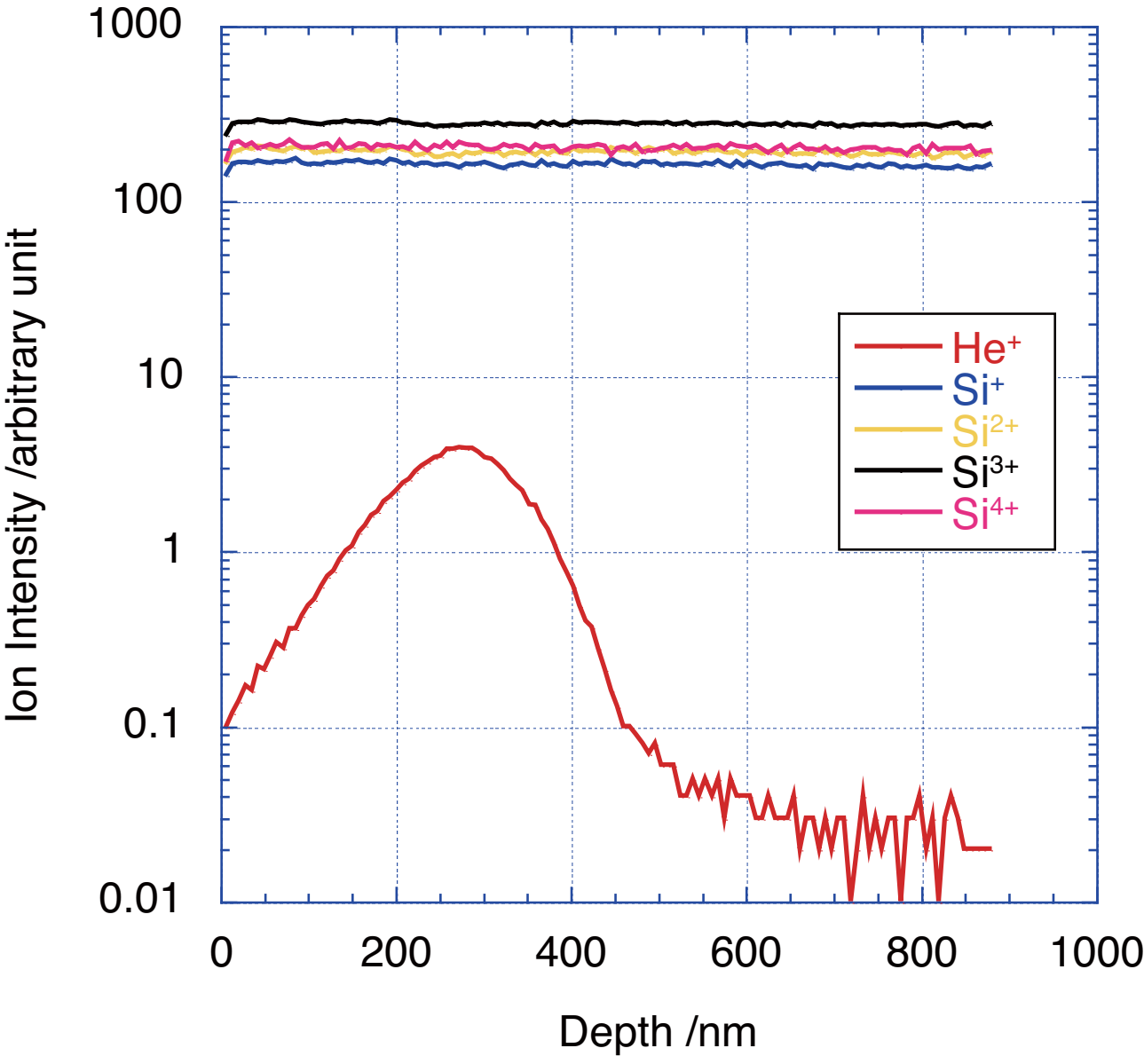




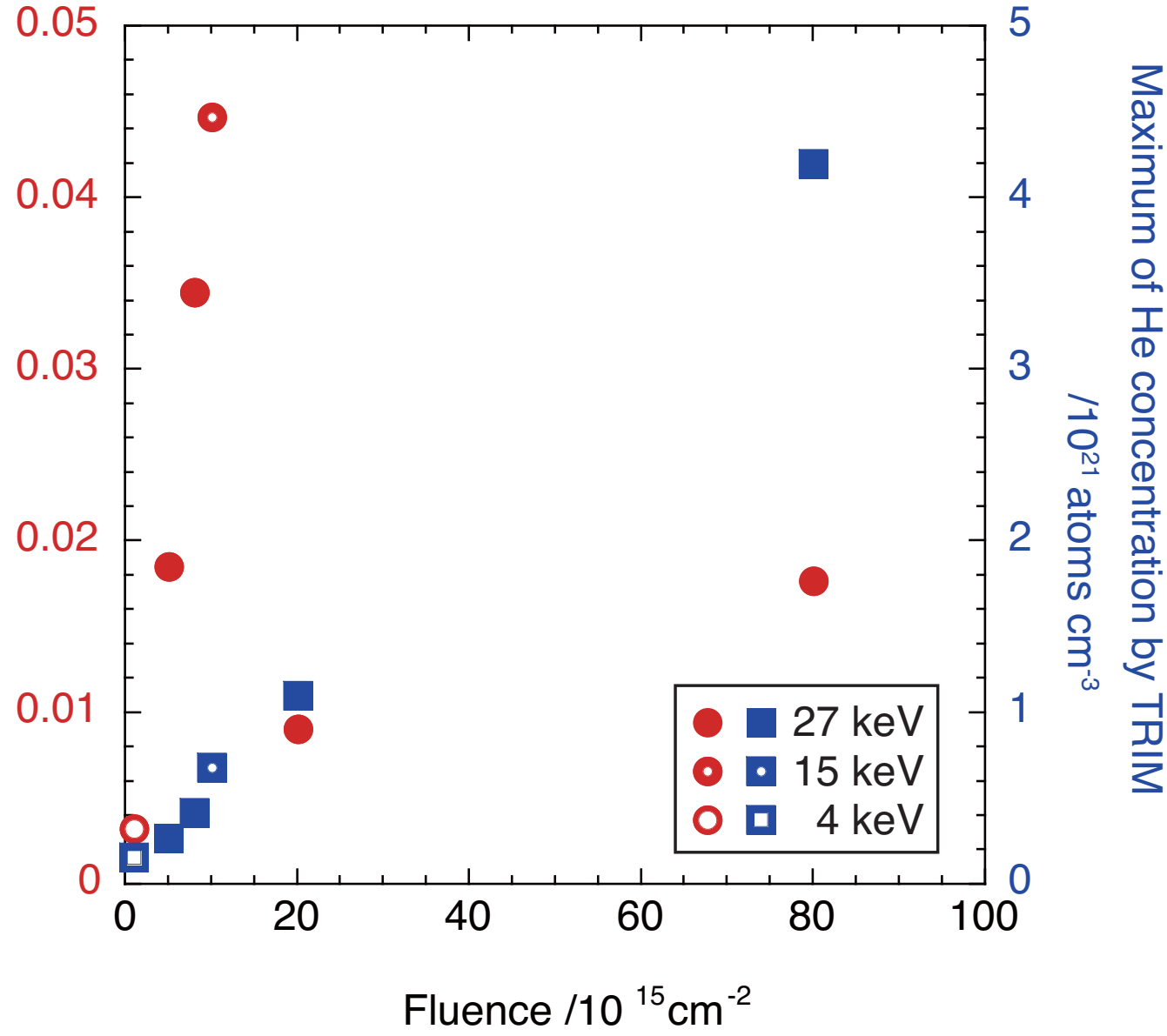




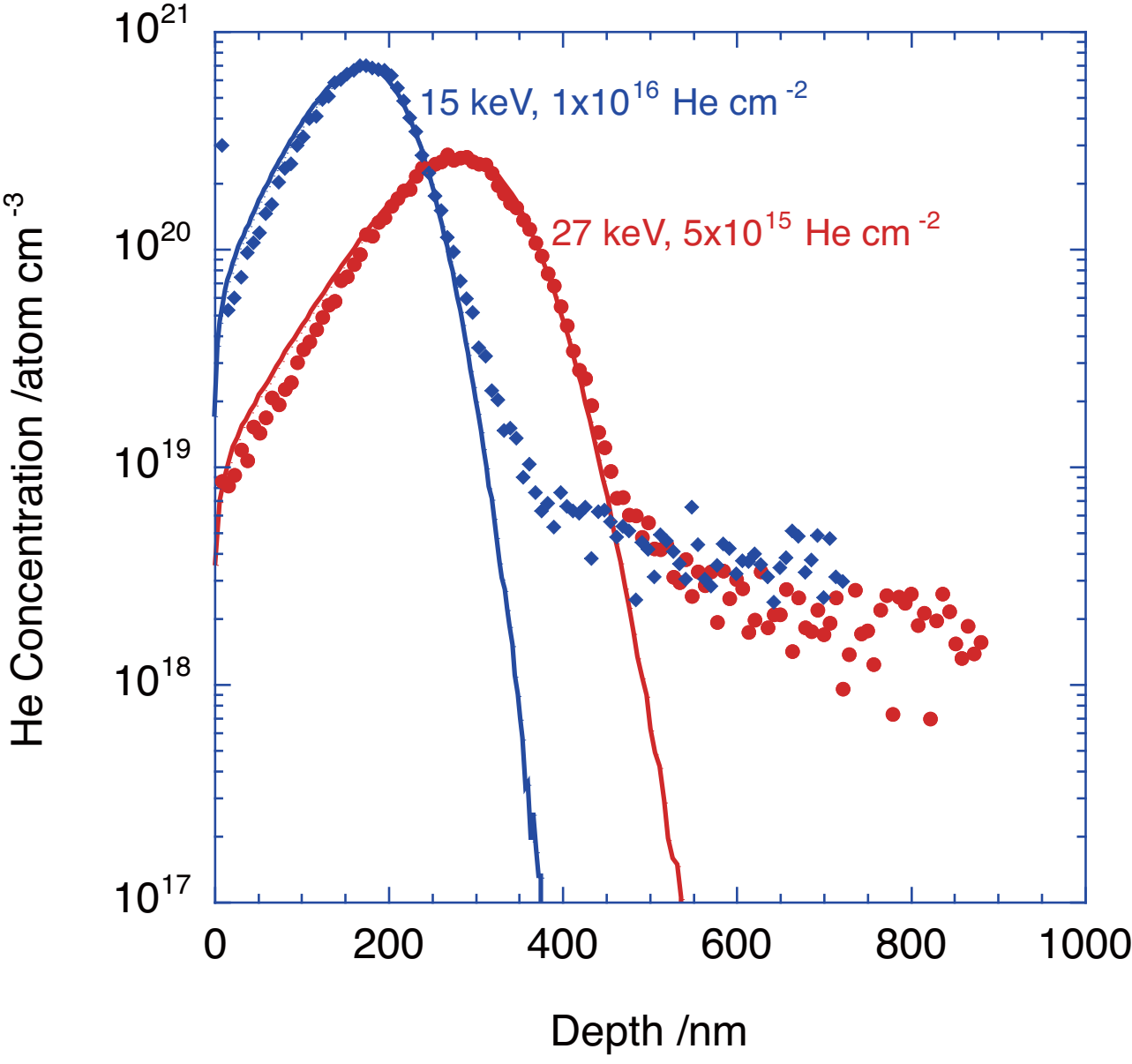




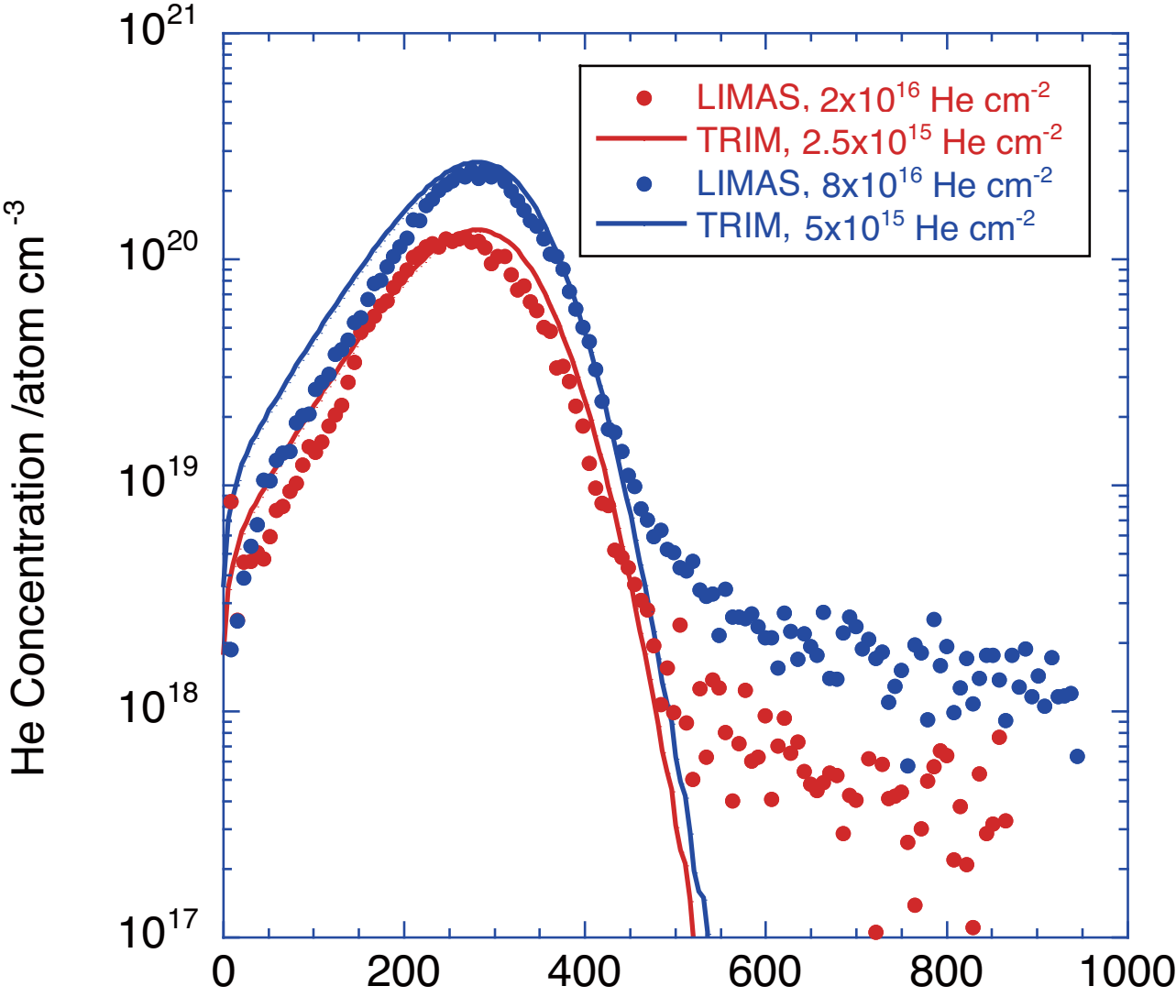

Depth 